\title{
Identification of Volatile Biomarkers of Gastric Cancer Cells and Ultrasensitive Electrochemical Detection based on Sensing Interface of Au-Ag Alloy coated MWCNTs
}

\author{
Yixia Zhang1, Guo Gao1, Huijuan Liu², Hualin Fu¹, Jun Fan³ ${ }^{3}$ Kan Wang1, Yunsheng Chen', Baojie Li², \\ Chunlei Zhang ${ }^{1}$, Xiao Zhi ${ }^{1}$, Lin $\mathrm{He}^{2}$, Daxiang Cui ${ }^{1,2}{ }^{\square}$ \\ 1. Institute of Nano Biomedicine and Engineering, Key Laboratory for Thin Film and Microfabrication Technology of the Ministry of Education, Research \\ Institute of Micro/Nano Science and Technology, Bio-X Center, Shanghai Jiao Tong University, Dongchuan Road 800, 200240 Shanghai, People's Republic \\ of China. \\ 2. Key Laboratory for the Genetics of Developmental \& Neuropsychiatric Disorders of Ministry of Education, Bio-X Center, Shanghai Jiao Tong University, \\ Dongchuan Road 800, 200240 Shanghai, People's Republic of China \\ 3. Department of Analytical Instruments, Shimadzu Shanghai Office, Room 201, Block G, No.570 West Huaihai Road, Shanghai 200052, P. R. China.
}

$\triangle$ Corresponding author: Daxiang Cui, Address: 800 Dongchuan Road, Shanghai 200240, People's Republic of China. E-mail: dxcui@sjtu.edu.cn.

( ) Ivyspring International Publisher. This is an open-access article distributed under the terms of the Creative Commons License (http://creativecommons.org/ licenses/by-nc-nd/3.0/). Reproduction is permitted for personal, noncommercial use, provided that the article is in whole, unmodified, and properly cited.

Received: 2013.09.02; Accepted: 2013.II.03; Published: 2014.0I.05

\begin{abstract}
Successful development of novel electrochemical biosensing interface for ultrasensitive detection of volatile biomarkers of gastric cancer cells is a challenging task. Herein we reported to screen out novel volatile biomarkers associated with gastric cancer cells and develop a novel Au-Ag alloy composites-coated MWCNTs as sensing interface for ultrasensitive detection of volatile biomarkers. MGC-803 gastric cancer cells and GES-I gastric mucous cells were cultured in serum-free media. The sample preparation approaches and HS-SPME conditions were optimized for screening volatile biomarkers. Volatiles emitted from the headspace of the cells/medium culture were identified using GC-MS. The Au-Ag nanoparticles-coated multiwalled carbon nanotubes were prepared as a sensing interface for detection of volatile biomarkers. Results showed that eight different volatile metabolites were screened out between MGC-803 cells and GES-I cells. Two compounds such as 3-octanone and butanone were specifically present in the headspace of the MGC-803 cells. Three volatiles such as 4-isopropoxybutanol, nonanol and 4-butoxy I-butanol coexisted in the headspace of both the MGC-803 cells and the GES-I cells, their concentrations in the headspace of the GES-I cells were markedly higher than those in the MGC-803 cells, three volatiles such as formic acid propyl ester, I.4-butanediol and 2, 6, II-trimethyl dodecane solely existed in the headspace of the GES-I cells. The nanocomposites of MWNTs loaded with Au-Ag nanoparticles were prepared as a electrochemical sensing interface for detection of two volatile biomarkers, cyclic voltammetry studies showed that the fabricated sensor could detect 3 -octanone in the range of $0 \sim 0.0025 \%(\mathrm{v} / \mathrm{v})$ and with a detection limitation of $0.3 \mathrm{ppb}$, could detect butanone in the range of $0 \sim 0.055 \%(\mathrm{v} / \mathrm{v})$, and with a detection limitation of $0.5 \mathrm{ppb}$, and exhibited good selectivity. The novel electrochemical biosensor combined with volatile biomarkers of gastric cancer owns great potential in applications such as early diagnosis and the prognosis of gastric cancer in near future.
\end{abstract}

Key words: gastric cancer cells; volatile organic compounds; multi-wall carbon nanotubes; Au-Ag nanoparticles; cyclic voltammetry; electrochemical sensor. 


\section{Introduction}

Stomach cancer is the fourth most common cancer and the second leading cause of cancer-related death in the world [1-3]. It remains very difficult to cure effectively, primarily because most patients present with advanced diseases [4]. Therefore, how to find early gastric cancer cells is a great challenge for early diagnosis and therapy of patients with gastric cancer. We have tried to establish an early gastric cancer warning system since 2005 [5], and hoped to find early gastric cancer cells in vivo by multi-mode targeted imaging techniques [6,-14]. However, the research was hampered by the lack of specific biomarkers to detect early gastric cancer. Searching for specific biomarkers of early gastric cancer has become our concern. One promising technology is the analysis of volatile metabolites that are released from cancer cells [15-19]. Volatile organic compounds (VOCs) that are emitted from cancer cell metabolism are considered to be important indicators for biochemical processes occurring in cancer cells [20,21]. Analysis of VOCs may be capable of prognosticating and diagnosing early cancer. Compared with genomics and proteomics, volatile metabolites embody pathway feedback mechanisms, which highly indicate the potential pathophysiological progress in cancer cells [22, 23]. To a certain extent, volatile metabolites represent the status of cancer cells. Looking for volatile biomarkers from gastric cancer cells and developing ultrasensitive detection method will be helpful for early warning and diagnosis of gastric cancer.

Recently, great advances in screening volatile biomarkers associated with cancer have been made. For example, common cancers such as lung cancer, breast cancer, melanoma, and colon carcinoma have been confirmed to exist specific volatile biomarkers [24, 25]. Peng, et al. reported a novel method to diagnose early lung cancer using gold nanoparticles combined with VOCs profiles [26]. Because of its non-invasive, easily collected samples, and easily repeating detection, this novel cancer diagnosis method based on VOCs profiles exhibit great potential in clinical applications. Buszewski, et al. investigated the volatiles emitted from gastric cancer tissues and cultured helcobacter pylori bacteria $(\mathrm{Hp})$, results showed that eighteen compounds were identified in stomach tissues, of which the amount of 1-propanol and carbon disulfide in the gaseous composition is higher in cancer tissue than in normal tissue, Hp bacteria present in the stomach might cause the increase in the concentration of 1-propanol and carbon disulfide in emission from cancer tissue $[27,28]$. However, so far few specific volatile biomarkers associated with early gastric cancer cells were reported. Therefore, looking for specific volatile biomarkers associated with early gastric cancer cells is still our focus.

Up to date, isolation and identification of biological VOCs are mainly performed by gas chromatography coupled with mass spectrometry (GC-MS), which can analyze multiple compounds simultaneously with a high sensitivity. However, this technique requires complicated procedures for detecting trace volatile compounds. Usually, samples are needed to pre-concentrate and extract with solid-phase microextraction (SPME) before GC-MS analysis [28, 29]. This process is time-consuming and the equipment is expensive. So the development of a non-invasive, quick and portable approach to detect VOCs has become a hotspot.

Electrochemical biosensors that utilize nanomaterials as building blocks offer a lot of advantages and have attracted the attention of many research groups. For example, Zhang, et al. fabricated a three-dimensional (3D) architecture by combining nitrogen-doped carbon nanotubes, thionine, and gold nanoparticles via a simple layer-by-layer method to evaluate cell surface carbohydrate and glycoprotein. This biosensor showed excellent analytical performance for the detection of HeLa cells with a lower detection limit of 500cells $\mathrm{mL}^{-1}$ [30]. Bali, et al. reported a dual-template imprinted polymer film containing dispersed multi-walled carbon nanotubes ceramic electrode was successfully used for ultra trace simultaneous analysis of ascorbic acid and dopamine, which was large enough to allow selective and sensitive analysis of one in the presence of other, without any cross reactivity, interferences and false-positives [31]. Espinosa, et al. reported that multi-walled carbon nanotubes (MWNTs) covered with gold or silver nanoclusters, as gas sensitive materials, could improve the selectivity of sensors [32,33]. We also prepared bio-mimetically synthesized Ag@BSA microspheres as a novel electrochemical biosensing interface, realized KB cells with a lower detection limit of 20cells $\mathrm{mL}^{-1}$ [34]. We also used self-assembly of multi-walled carbon nanotubes and quantum dots to realize the ultrasensitive detection of DNA and antigen [35]. These examples fully demonstrate using carbon nanotubes and noble metal nanoparticles to develop ultrasensitive electrode interfaces is a good design strategy for fabricating new generation of electrochemical biosensors, because the nanocomposites of CNTs/nobel metal nanoparticles could enhance the performance of electrochemical biosensors [36, 37], for example, to improve the sensitivity of electrochemical sensors and to increase electron-transfer resistance because of their nanostructure and large surface area [38-40]. In recent years, nobel metal nanoparticles, especially bimetallic nanoparticles, for example, 
$\mathrm{Au}-\mathrm{Ag}$ alloy nanoparticles, because of synergistic catalytic abilities, and selectivity of electrochemical reactions, exhibit great potential in development of new generation of electrochemical biosensors [41-43].

In this study, we report that the potential volatile biomarkers associated with gastric cancer cells were identified by SPME/GC-MS, using advantages of carbon nanotubes and nobel metal nanoparticles, a new electrochemical sensing interface, composed of $\mathrm{Au}-\mathrm{Ag}$ alloy nanoparticles coating multi-walled carbon nanotubes (MWNTs) were prepared, two volatile biomarkers of gastric cancer cells screened were used as the detection targets, a new electrochemical biosensor detection system with high sensitivity and selectivity was developed. The identified volatile biomarkers of gastric cancer cells and established electrochemical biosensor may have great potential in applications such as early screening and the pre-warning of gastric cancer in near future.

\section{Materials and Methods}

\section{Cell culture}

MGC-803 gastric cancer cell line and GES-1 gastric mucosa cell line were cultured in RPMI 1640 media containing $5 \%$ heat-inactivated fetal bovine serum. The cells were detached using trypsin and harvested by centrifuging at $1200 \mathrm{rpm} / \mathrm{min}$ for 3 minutes, and then they were counted with a hemacytometer and reconstituted with fresh media and were seeded into a $75 \mathrm{~cm}^{2}$ sealed cell culture flask with $40 \mathrm{~mL}$ of serum-free cell media at a density of approximately $1 \times 10^{6}$ cells $/ \mathrm{mL}$. The flasks were incubated at $37{ }^{\circ} \mathrm{C}$ for the desired time in a humidified atmosphere of $5 \% \mathrm{CO}_{2}(\mathrm{~V} / \mathrm{V})$ with a control group that contained the same volume of media without cells, reaching a typical confluence of $70 \sim 80 \%$.

\section{Apparatus and SPME Extraction}

The VOCs from cell metabolism and cell-free media were extracted and pre-concentrated by HS-SPME (headspace solid-phase microextraction). 20 $\mathrm{mL}$ airtight vials were thoroughly cleaned, sterilized and baked at $120^{\circ} \mathrm{C}$ for 20 hours before use. $10 \mathrm{~mL}$ of medium with cells cultured for 22 hours was transferred from the flasks to HS vials and sealed tightly with a PTFE septum. The cell free media that was subjected to identical conditions was set as the experimental control. A 57330-U manual holder and 75 $\mu \mathrm{m}$ CAR/PDMS coating was used for the HS sampling of the VOCs. Before the first use, the SPME fiber was conditioned in a GC injector at $280^{\circ} \mathrm{C}$ for 1 hour. The blank desorption of the fiber was performed under identical oven temperatures to clear localized background influences. Next, sampling was performed by introducing the SPME fiber into the head- space of the glass HS vial containing cell media samples through a PTFE septum in a hermetically sealed cap and exposing it for $44 \mathrm{~min}$ in a $38^{\circ} \mathrm{C}$ water bath. A teflon stir bar was used and driven by a magnetic stirrer. To avoid the condensation of released VOCs, the parts outside the water bath were kept at $38^{\circ} \mathrm{C}$. All the vials were thermostated at $38^{\circ} \mathrm{C}$ for $10 \mathrm{~min}$ before SPME sampling. After sampling, the fiber was desorbed in a GC injector at $280^{\circ} \mathrm{C}$ within 2 min splitless times at a split ratio of 1:20. Helium was used as a carrier gas with a linear velocity of $44.2 \mathrm{~cm} \mathrm{~s}^{-1}$. The analysis was performed on a GC/MS (QP2010E, Shimadzu). The separation and detection were performed on a $30 \mathrm{~m} \times 0.25 \mathrm{~mm} \times 0.25 \mu \mathrm{m}$ Rxi-5 ms capillary column. The oven temperatures were as follows: initial $40{ }^{\circ} \mathrm{C}$, held for $5 \mathrm{~min}$ and then ramped at $10{ }^{\circ} \mathrm{C}$ $\mathrm{min}^{-1}$ to $260^{\circ} \mathrm{C}$ and held for $10 \mathrm{~min}$. The MS analyses were conducted in a full-scan mode, with a scan range of 42-400 a.m.u. The electron impact ionization was used at an energy of $70 \mathrm{eV}$. The temperatures of the ion source and the quadrupole were $200{ }^{\circ} \mathrm{C}$ and 250 ${ }^{\circ} \mathrm{C}$, respectively.

\section{Fingerprint of VOCs for various cell lines}

A comparison of volatile biomarkers between the MGC-803 and the GES-1 cells was performed. The different substances were identified by spectral match with the National Institute of Standards and Technology (NIST) 2008 MS spectral library (Gatesburg, PA, USA). To ensure the maximum reliability of the results, the identification was performed not only by spectral library match using the NIST 08 library, but was also verified by confirmation of the retention time for compounds with a similarity coefficient over $85 \%$. The BRENDA database was used for the initial correlation between the detected compounds and the metabolic pathways [44].

\section{Preparation and characterization of MWNTs loaded on Au-Ag alloy nanoparticles}

An acid-treated MWNTs water solution (0.1 $\mathrm{mg} / \mathrm{mL}, \mathrm{pH}=7.5$ ) was provided by Qifa Liu, Ph.D from Shanghai Jiao Tong University. $1 \mathrm{~mL}$ of 0.1 $\mathrm{mg} / \mathrm{mL}$ MWNTs water solution was added to a 20 $\mathrm{mL}$ vial, followed by the addition of $1 \mathrm{~mL}$ of $10 \mathrm{mM}$ $\mathrm{HAuCl}_{4}$ solution and $1 \mathrm{~mL}$ of $10 \mathrm{mM} \mathrm{AgNO}$ solution. The reaction mixture was adjusted to $15 \mathrm{~mL}$ with ultrapure water and stirred continuously at $25^{\circ} \mathrm{C}$ for $12 \mathrm{~h}$ for MWNTs that were loaded on Au-Ag nanoparticles. The MWNTs that were decorated with Au-Ag nanoparticles were studied by Scanning Electron Microscopy (SEM), transmission electroscopy (TEM) and Energy-dispersive X-ray spectroscopy (EDS) analysis. For comparison to MWNTs/Au-Ag alloy nanocomposites, MWNTs loaded with Ag nanoparticles or Au 
nanoparticles were prepared respectively, $2 \mathrm{~mL}$ MWNTs water solution $(0.1 \mathrm{mg} / \mathrm{mL}, \mathrm{pH}=7.0)$ was added to vessels followed by the addition of $2 \mathrm{~mL}$ of $10 \mathrm{mM} \mathrm{AgNO} 3$ or $2 \mathrm{~mL}$ of $10 \mathrm{mM} \mathrm{HAuCl} 4$ solution. The mixture was adjusted to $15 \mathrm{~mL}$ and stirred at $25^{\circ} \mathrm{C}$ for $12 \mathrm{~h} \mathrm{[45-48].}$

\section{Fabrication of the electrochemical biosensor}

The glass carbon electrode (GCE) was polished with $0.03 \mu \mathrm{m}$ alumina powder on a micro-cloth and then thoroughly cleansed ultrasonically with ethanol and doubly distilled water, respectively. The clean glass carbon electrode was dried with $\mathrm{N}_{2}$. The clean glass carbon electrode was dried with $\mathrm{N}_{2} .20 \mu \mathrm{L}$ of MWNTs/Au-Ag alloy composite solution were dropped onto the surface of the GCE and air dried.

\section{Electrochemical measurements}

All the electrochemical experiments were performed with a CHI660D electrochemical workstation ( $\mathrm{CH}$ Instruments, USA) using a conventional three-electrode cell at room temperature. The working electrodes, CHI150 saturated calomel electrode (SCE) and a platinum wire, were used for reference and auxiliary electrodes, respectively. The modified electrodes were positioned in $20 \mathrm{~mL}$ of $0.1 \mathrm{M} \mathrm{KCl}$ solution. For the deoxygenated experiments, $\mathrm{KCl}$ solution was bubbled with high-purity nitrogen for $15 \mathrm{~min}$, and the nitrogen condition was maintained during the experiments. A cyclic voltammetric scan $(-0.5 \sim 0.5 \mathrm{~V})$ was applied to the working electrode until the current maintained a steady state.

The electrochemical test was described as follows: the steady MWNTs/Au-Ag/GCE was put in the headspace of samples for $45 \mathrm{~min}$ to adsorb the volatile biomarkers. Then, MWNTs/Au-Ag/GCE put in the three-electrode cell. In order to compare with
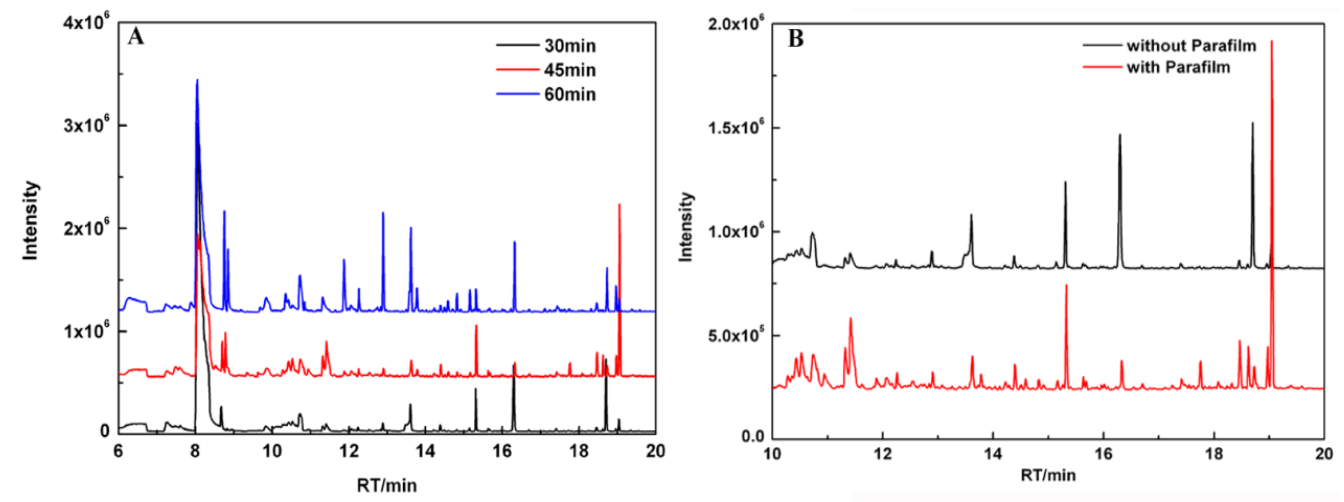

Figure I. The patterns from the chromatogram of VOCs from MGC-803 gastric cancer cells that were influenced by: (A) headspace extraction time; (B) MGC-803 cells cultured with and without Parafilm.
MWNTs/Au-Ag/GCE, MWNTs/Ag/GCE, MWNTs / Au/GCE, MWNTs/GCE and bare GCE were set as control. Finally, the electrochemical test was carried out in $0.1 \mathrm{M} \mathrm{KCl}$ at $60 \mathrm{mV} / \mathrm{s}$ from -0.5 to $0.5 \mathrm{~V}$.

\section{Statistical Analysis}

All the data are presented in this paper as the mean result \pm S.D. Significant differences were evaluated using the $t$-test and considered significant at $P<$ 0.05 .

\section{Results and Discussion}

\section{Identification of volatile biomarkers associated with gastric cancer cells}

The influence of headspace pre-concentration time by SPME on the chromatograms of cultured cells was investigated. To obtain better chromatograms, different extracting times ( $30 \mathrm{~min}, 45 \mathrm{~min}$, and $60 \mathrm{~min}$ ) were used to pre-concentrate volatiles in the headspace of samples. As shown in Fig. 1A, the optimal pre-concentration time of HS-SPME was $45 \mathrm{~min}$, which was strongly preferred to perform the GC-MS analysis in our study. Besides, these volatiles are time-dependent and occur in very low concentrations. Different incubation time (12 h, $24 \mathrm{~h}$, and $36 \mathrm{~h}$ ) were used in our study. Meanwhile, cell culture flasks were protected with Parafilm. It was observed that significant increase in the emission of VOCs at $24 \mathrm{~h}$. We found that cell viability decreased with incubation time. Cell culture flasks protected with Parafilm could substantially improve the chromatogram profile of volatile metabolites (Fig. 1B). Under optimized conditions, eight different volatile compounds between MGC 803 cells and GES-1 cells were identified reproducibly and summarized in Table 1. 
Table I. Summary of different VOCs between MGC-803 cells and GES-I cells.

\begin{tabular}{lllll}
\hline Peak & RT & Compounds & GES-1 & MGC-803 \\
\hline 1 & 9.1 & Formic acid propyl ester & + & - \\
2 & 10.4 & 3-octanone & - & + \\
3 & 10.8 & 1.4-butanediol & + & - \\
4 & 11.6 & 4-Isopropoxybutanol & ++ & + \\
5 & 12.75 & nonanol & ++ & + \\
6 & 18.08 & Butanone & - & + \\
7 & 18.32 & 1-butanol,4-butoxy & ++ & + \\
8 & 18.84 & Dodecane, 2,6,11-trimethyl & + & - \\
\hline
\end{tabular}

Notes: +: VOCs present; -: VOCs absent; different numbers of + stands for different concentrations.

Fig.2 is a representative chromatogram illustrating different volatiles in the headspace from the MGC-803 cells, the GSE- 1 cells and the culture medium. Among the eight different compounds (peak 1-peak 8), peak 2 (Fig. 2A) and peak 6 (Fig. 2B) were only present in the headspace of the MGC-803 cells. Further analysis identified them as 3-octanone and butanone. Additionally, three compounds, formic acid propyl ester (peak 1), 1.4-butanediol (peak 3) and dodecane, 2, 6, 11-trimethyl (peak 8), solely existed in the headspace of the GES-1 cell media.

Compounds such as 4-Isopropoxybutanol (peak 4), nonanol (peak 5) and 1-butanol, 4-butoxy (peak 7) were higher concentrations in the headspace of the GES-1 cells compared to the MGC- 803 cells. The ratio of the relative peak area of them between the GES-1 cells and the MGC-803 cells were 4-Isopropoxybutanol $\leq 0.3$, nonanol $\leq 0.36$ and 1-butanol (4-butoxy) $\leq 0.40$.

We firstly identified volatile biomarkers to distinguish MGC-803 gastric cancer cells from GES-1 gastric mucous cells by HS-SPME/GC-MS. We at-

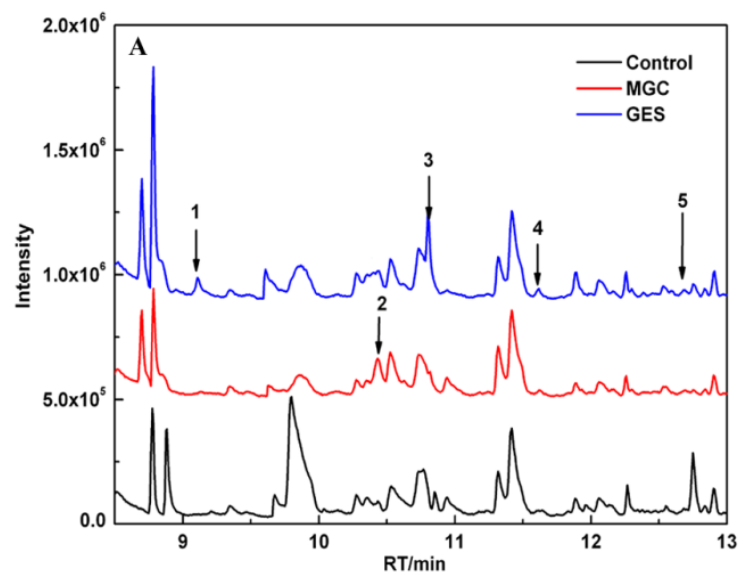

tempted to optimize the methods of preparation and pre-concentration of samples. It is well known that the majority of VOCs are intermediate products of cell metabolism. So the cell culture period has a significant influence on the profile of VOCs chromatograms. Furthermore, biological VOCs are volatile with very low concentrations. The cell culture flask protected with Parafilm can avoid the omission of volatile biomarkers and environmental interferences. Therefore, the culture time and culture conditions were optimized in this work. Under the optimal conditions, with the same headspace-to-liquid volume ratio, the magnitude of the extracting effect of the VOCs was larger than that with lower headspace volume. According to the protocol reported [27-29], $10 \mathrm{~mL}$ of cell media was introduced into $20 \mathrm{~mL}$ of vial headspace for HS-SPME. Furthermore, extraction time is also an important factor. The shorter sampling time $(30 \mathrm{~min})$ caused the lower extractable absorption of analytes as a result of the reduced sensitivity of SPME. The longer HS pre-concentration time (60 min) enabled competitive absorption, which could result in a lower efficiency for extracting compounds and some valuable biomarkers may be lost. Finally, a 45 min HS-SPME sampling time was strongly preferred in this work and showed good sensitivity.

Our data showed that the amount of alcohols or aldehydes in the headspace of the MGC-803 cells were higher than those in the GES-1 cells. These phenomena should be attributed to the higher activity of aldehyde dehydrogenase in cancer cells [48-50]. Alcohols or aldehydes were oxidized to the corresponding carboxylic acids in an alcohol dehydrogenase-independent pathway; carboxylic acids were further metabolized as precursors of the synthesis of cell membranes, which provide more substances to meet the rapid growth requirement of cancer cells.

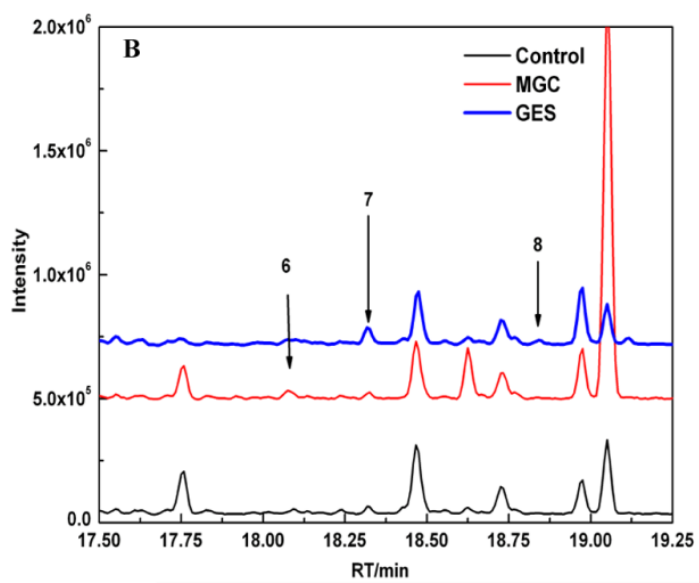

Figure 2. Comparison of VOCs chromatograms from the headspace of the MGC-803 cells, the GES-I cells and media culture: (A) the chromatograms with a retention time 8 /3 min; (B) the chromatograms with a retention time 17 20 min. 


\section{Characterization of MWNTs/Au-Ag alloy nanocomposites}

The nanocomposite of MWNTs/Au-Ag nanocomposites was successfully prepared. The MWNTs/Au-Ag composites were further characterized by TEM and SEM (Fig. 3A and 3B). The TEM and SEM images indicated that $\mathrm{Au}-\mathrm{Ag}$ nanoparticles were loaded on the surface of MWNTs. The size and micro-structure of Au-Ag nanoparticles loaded on the surface of MWNTs was examined by high resolution transmission electroscopy (HR-TEM) in Fig. 3C. The HR-TEM images showed that the Au-Ag nanopartcles were spherical with diameters of 20 25 $\mathrm{nm}$. HR-TEM also confirmed the formation of homogeneous gold-silver alloy nanocrystals, rather than either a segregated metal or a core/shell structure. EDS analysis was conducted and further confirmed that the composites comprised of carbon, gold and silver elements, as shown in Fig. 3D.

\section{Electrochemical behavior of MWNTs/Au- Ag/GCE}

The electrochemical behaviors of different modified electrodes (MWNTs/Au-Ag/GCE, MWNTs/Ag /GCE, MWNTs/Au/GCE, MWNTs/GCE and bare GCE) in $0.1 \mathrm{M} \mathrm{KCl}$ at $60 \mathrm{mV} / \mathrm{s}$ from -0.5 to $0.5 \mathrm{~V}$., and the CVs were shown in Fig. 4A. A pair of well-defined redox peaks was observed on MWNTs/Au-Ag/GCE. The current response of MWNTs/Au-Ag/GCE ( $\mathrm{I}_{\mathrm{p}}=$ $602.9 \mu \mathrm{A}, \mathrm{E}_{\mathrm{p}}=-116 \mathrm{mv}$ ) is nearly 26 times stronger than that of MWNTs/Ag/GCE $\left(\mathrm{I}_{\mathrm{p}}=23.21 \mu \mathrm{A}, \mathrm{E}_{\mathrm{p}}=-176\right.$ $\mathrm{mv})$, is far away stronger than that of MWNTs/Au/GCE. This suggested that the MWNTs/Au-Ag nanocomposites, as electrode sensing film, exhibited evident advantages over other nanomaterials. The phenomenon could be explained as follows: firstly, Au-Ag alloy with large surface area could enable fast electron-transfer [45]. Secondly, MWNTs are known to not only can selectively adsorb gas molecules but also increase the surface area for $\mathrm{Au}-\mathrm{Ag}$ alloy adsorption. So the better electronic conductive characteristics of MWNTs/Au-Ag/GCE were mainly attributed to the synergistic effect between the MWNTs and the Au-Ag nanoparticles.

The CV curves of MWNTs/Au-Ag/GCE with different scan rates $(20 \mathrm{mv}-200 \mathrm{mV} / \mathrm{s})$ are obtained in Fig. 4B. The cathodic peak currents of MWNTs/Au-Ag/GCE were directly proportional to the scan rates at a range of $20-200 \mathrm{mV} / \mathrm{s}$ (the inset of Fig. $4 \mathrm{~B} ; \mathrm{I}_{\mathrm{p}}=3.5035 \mathrm{x}+144.51, \mathrm{R}=0.9909$ ). This indicated that it is an adsorption-controlled process on the surface of MWNTs/Au-Ag/GCE.

\section{Electrochemical test of butanone and 3-octanone on MWNTs/Au-Ag/GCE}

The electrochemical sensor was used to respectively detect different concentrations of butanone and 3-octanone in $\mathrm{N}_{2}$ matrix. With the concentration of butanone increasing, a gradual decrease of the anodic peak current $\left(\mathrm{I}_{\mathrm{p}}\right)$ was observed with a negative shift of potential (Fig. 5A). The $\mathrm{I}_{\mathrm{P}}$ is linear in the concentration of butanone $(C)$ range between 0 and $0.55 \mathrm{ppm}$ with the detection of limitation (LOD) of $0.5 \mathrm{ppb}(\mathrm{S} / \mathrm{N}=3)$. The regression equation (shown in the inset of Fig. $5 \mathrm{~A})$ is $\mathrm{I}_{\mathrm{p}}=-9.8886 \mathrm{C}+445.92$ with a correlation coefficient (r) 0.9986. At the same detection of 3-octanone was performed in Fig. 6B. The $\mathrm{I}_{\mathrm{p}}$ also exhibited a good linear relationship with the concentrations of 3 -octanone $(C)$ in the range of $0 \sim 0.25 \mathrm{ppm}$ with the LOD of $0.3 \mathrm{ppb}(\mathrm{S} / \mathrm{N}=3)$. The regression equation (shown in the inset of Fig. 5B) is $\mathrm{I}_{\mathrm{p}}=$ $-18.8857 C+309.0143$ with $r$ of 0.9956 . It was observed that the electrochemical assay system can sensitively detect butanone and 3-octanone.

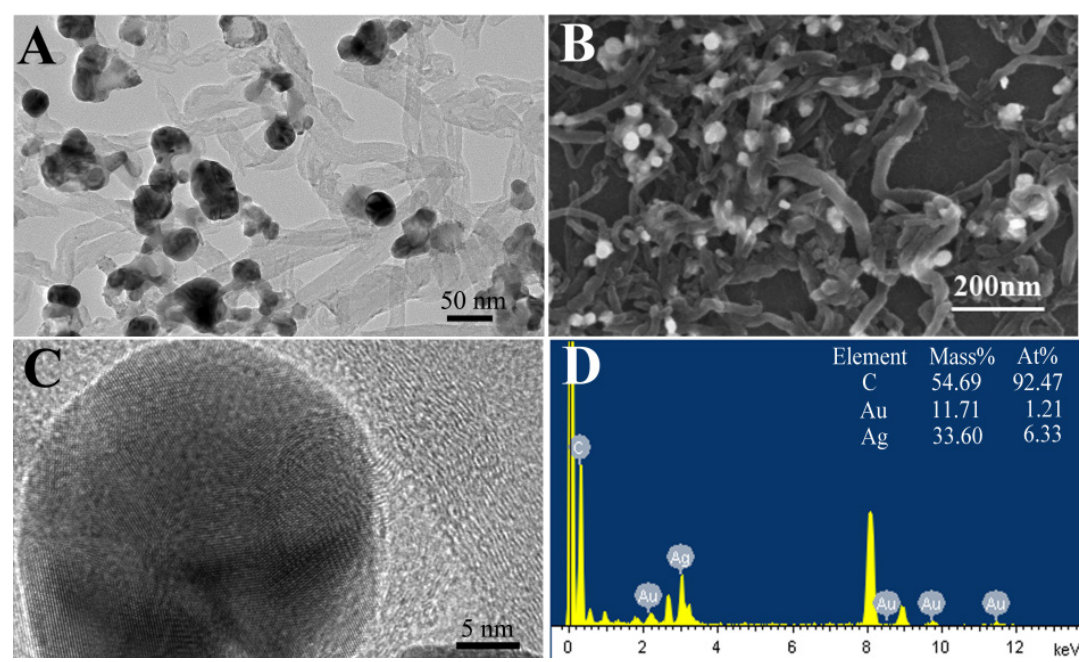

Figure 3. Characterization of MWNTs covered with Au-Ag nanoparticles: (A) TEM image; (B) SEM image; (C) HR-TEM image of Au-Ag nanoparticles on the surface of MWNTs; (D) the corresponding EDX spectra of MWNTs/Au-Ag nanoparticles. 

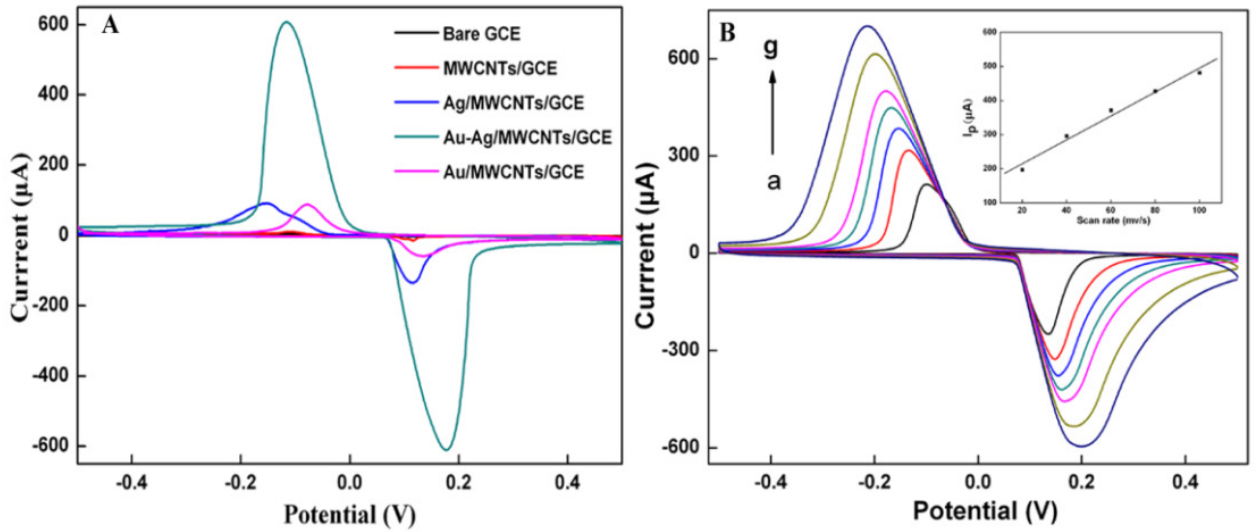

Figure 4. (A) Cyclic voltammograms of MWNTs/Au-Ag/GCE, MWNTs/GCE, MWNTs/Ag/GCE, MWNTs/Au/GCE and bare GCE in 0.I M KCl solution. (B) Cyclic voltammograms of MWNTs/Au-Ag/GCE in $0.1 \mathrm{M} \mathrm{KCl}$ solution with various scan rates (from a to g: 20,40,60,80, 100,150 and 200, respectively). The inset is the linear plot between the anodic peak current and the scan rate.
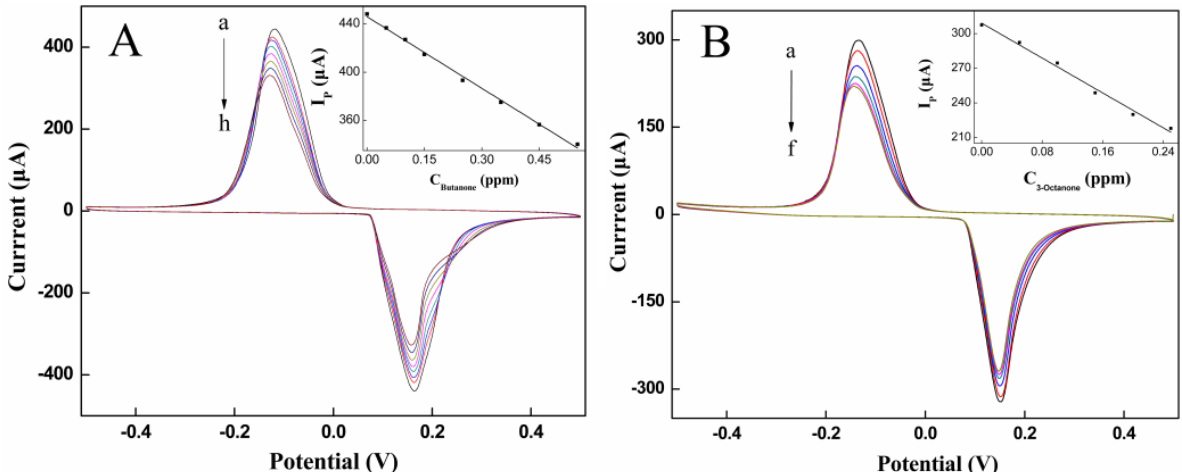

Figure 5. (A): The cyclic voltammograms of MWNTs/Au-Ag/GCE were exposed to different concentrations of butanone (from a to $\mathrm{h}: 0,0.05,0.1,0.15$, $0.25,0.35,0.45$ and $0.55 \mathrm{ppm}$, respectively). The inset shows a line plot of the peak current $\left(\mathrm{I}_{\mathrm{p}}\right)$ against concentration of butanone. (B): The cyclic voltammograms of MWNTs/Au-Ag/GCE were exposed to different concentrations of 3-octanone (from a to f: 0, 0.05, 0.1, $0.15,0.2$ and 0.25 ppm, respectively). The inset shows a line plot of the peak current $\left(I_{p}\right)$ against concentration of 3-octanone. All the potentials are given versus SCE.

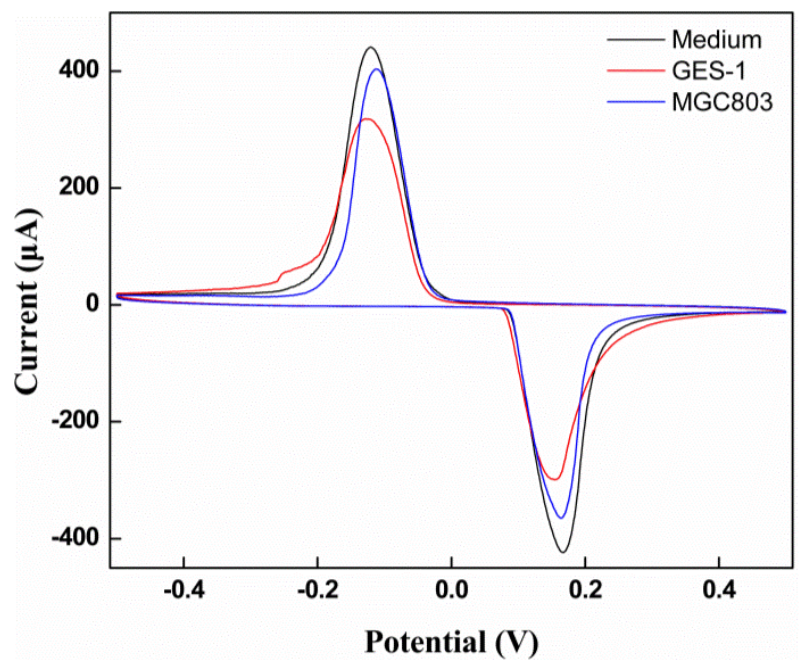

Figure 6. Cyclic voltammograms of MWNTs/Au-Ag/GCE was exposed to the head space of cell-free medium, MGC-803 gastric cancer cells, and GES-I gastric mucosa cells.

\section{Cell samples analysis}

The electrochemical assay system was used in real cell sample analysis to verify it the potential ap- plication [50]. MGC-803 gastric cancer cell released specific volatiles in the process of metabolism. Theses volatile biomarkers have electrochemical activity on the MWNTs/Au-Ag/GCE. Fig. 6 depicts the CVs of volatiles in the head space of MGC-803 gastric cancer cells, GES-1gastric mucosa cells, and cell-free medium.

When the MWNTs/Au-Ag/GCE was exposed to the head space of gastric cancer cell MGC-803, the peak potential moves to high potential with slightly decrease of peak current relatively to cell-free medium. However, compared with gastric cancer cell MGC-803, the peak current obviously decrease when MWNTs/Au-Ag/GCE was exposed to the head space of gastric mucosa cell GES-1 with no shift of peak potential. The results might be explained as follows: the butanone and 3-octanone had more electrochemical activity compared to other compounds shown in Table 1. According to the curves of CVs, MGC-803 gastric cancer cells can be easily discriminated from GES-1 gastric mucosa cells. This result foresees the promising application of MWNTs/Au-Ag nanocomposite as electrocatalyst in sensing and detection of 
MGC-803gastric cancer cells.

In order to investigate the cross-interference, we used $\mathrm{CO}_{2}$, acetone and ethanol as interference substances. No obvious change of the peak currents of 3-octanone and butanone was observed, suggesting that this electrochemical assay system based on MWNTs/Au-Ag/GCE has good selectivity.

\section{Conclusions}

In conclusion, it is the first report to identify volatile biomarkers to distinguish MGC-803 gastric cancer cells from GES-1 gastric mucous cells with HS-SPME coupling with GCMS. An electrochemical assay system based on MWNTs/Au-Ag/GCE was developed to detect the volatile biomarkers associated with gastric cancer cells with high sensitivity, selectivity and no cross-interference. The specific volatile biomarkers of MGC-803 gastric cancer cells and the well-adapted electrochemical system have considerable potential in the near future for applications, such as screening and warning of early gastric cancer. Further work will focus on developing new generation of electrochemical biosensors for simultaneous detection of more VOCs biomarkers.

\section{Acknowledgements}

This work is supported by the National Key Basic Research Program (973 Project) (No. 2011CB933100), National Natural Scientific Fund (No.81225010, 81327002, and 31100717), 863 project of China (2012AA022703), Shanghai Science and Technology Fund (No.13NM1401500 and 11nm0504200), Shanghai Jiao Tong University Innovation Fund for Postgraduates (No. AE340011).

\section{Competing Interests}

The authors have declared that no competing interest exists.

\section{References}

1. Jemal A, Siegel R, Ward E, et al. Cancer statistics, 2008. CA: a cancer journal for clinicians. 2008; 58: 71-96.

2. Bondy M. Cancer epidemiology and prevention. JAMA. 2009; 301: 1074

3. Xu AG, Li SG, Liu JH, et al. Function of apoptosis and expression of the proteins Bcl-2, p53 and C-myc in the development of gastric cancer. World J Gastroenterol. 2001; 7: 403-06.

4. Wang K, Ruan J, Qian Q, et al. BRCAA1 monoclonal antibody conjugated fluorescent magnetic nanoparticles for in vivo targeted magnetofluorescent imaging of gastric cancer. J Nanobiotechnol. 2011; 9: 23.

5. Cui DX, Zhang L, Yan XJ, et al. A microarray-based gastric carcinoma prewarning system. World J Gastroenterol. 2005; 11: 1273-82.

6. Kong Y, Chen J, Gao F, et al. A multifunctional Ribonuclease-A-conjugated CdTe Quantum Dot cluster nanosystem for synchronous cancer imaging and therapy. Small 2010; 6: 2367-73.

7. He M, Huang P, Zhang CL, et al. Dual phase-controlled synthesis of uniform lanthanide-doped $\mathrm{NaGdF}_{4}$ upconversion nanocrystals via an $\mathrm{OA}$ /ionic liquid two-phase system for in vivo dual-modality imaging. Advanced Functional Materials 2011; 21: 4470 - 4477.

8. Gao G, Zhang CL, Zhou ZJ, et al. One-pot hydrothermal synthesis of lanthanide ions doped one-dimensional upconversion submicrocrystals and their potential application in vivo CT imaging. Nanoscale 2013; 5: 351-362.
9. Huang P, Lin J, Wang XS, et al. Light-triggered theranostics based on photosensitizer-conjugated carbon dots for simultaneous enhanced-fluorescence imaging and photodynamic therapy. Adv Mater. 2012; 24: 5104-5110.

10. Ruan J, Song H, LI C, et al. DiR-labeled Embryonic Stem Cells for Targeted Imaging of in vivo Gastric Cancer Cells. Theranostics 2012; 2:618-628.

11. Wang K, Ma J, He M, et al. Toxicity Assessments of Near-infrared Upconversion Luminescent LaF3:Yb,Er in Early Development of Zebrafish Embryos. Theranostics 2013; 3: 258-266.

12. Zhi $X$, Liu $Q$, Zhang $X$, et al. Quick genotyping detection of HBV by giant magnetoresistive biochip combined with PCR and line probe assay. Lab on A Chip 2012; 12(4):741-745.

13. Huang P, Xu C, Lin J, et al. Folic Acid-conjugated Graphene Oxide loaded with Photosensitizers for Targeting Photodynamic Therapy. Theranostics 2011; 1:240-250.

14. Gao G, Wu HX, Gao WJ, et al. Preparation of $\mathrm{FeCO}_{3}-\mathrm{Fe}_{3} \mathrm{O}_{4}$ nanoparticles and flower-like assemblies via a one-step hydrothermal method. Crys-14 Eng Comm. 2011; 13: 6950-6954.

15. Miekisch W, Schubert JK, Noeldge-Schomburg GFE. Diagnostic potential of breath analysis--focus on volatile organic compounds. Clin Chim Acta. 2004; 347: 25-39.

16. Dubowski KM. Breath analysis as a technique in clinical chemistry. Clin Chem. 1974; 20: 966

17. Bartolazzi A, Santonico M, Pennazza G, et al. A sensor array and GC study about VOCs and cancer cells. Sensor Actuat B-Chem. 2010; 146: 483-88

18. Chen $\mathrm{X}, \mathrm{Xu} \mathrm{F}$, Wang $\mathrm{Y}$, et al. A study of the volatile organic compounds exhaled by lung cancer cells in vitro for breath diagnosis. Cancer 2007; 110: 835-44.

19. Burke D, Halpern B, Malegan D, et al. Profiles of urinary volatiles from metabolic disorders characterized by unusual odors. Clin Chem. 1983; 29: 1834-38.

20. Amann A, Poupart G, Telser S, et al. Applications of breath gas analysis in medicine. Int J Mass Spectrom. 2004; 239: 227-33.

21. Nicholson JK, Wilson ID. Understanding 'global' systems biology: metabonomics and the continuum of metabolism. Nat. Rev. Drug. Discov. 2003; 2: 668-76.

22. Modak AS. Breath Tests for Evaluating Enzyme Activity in Personalized Medicine. Drug Metab Rev. 2007; 39: 95-99.

23. Phillips M, Cataneo RN, Saunders C, et al. Volatile biomarkers in the breath of women with breast cancer. J Breath Res. 2010; 4: 026003.

24. Zhang XQ, Guo Q, Cui DX. Recent Advances in Nanotechnology Applied to Biosensors. Sensors 2009; 9: 1033-1053

25. Peng G, Hakim M, Broza YY, et al. Detection of lung, breast, colorectal, and prostate cancers from exhaled breath using a single array of nanosensors. Brit J Cancer. 2010; 103:542-551.

26. Peng G, Tisch U, Adams O, et al. Diagnosing lung cancer in exhaled breath using gold nanoparticles. Nat Nanotechnol. 2009; 4: 669-673.

27. Buszewski B, Ulanowska A, Ligor $\mathrm{T}$, et al. Identification of volatile organic compounds secreted from cancer tissues and bacterial cultures. J Chromatogr B. 2008 ; 868: 88-94.

28. Ligor T, Szeliga J, Jackowski M, et al. Preliminary study of volatile organic compounds from breath and stomach tissue by means of solid phase microextraction and gas chromatography mass spectrometry. J Breath Res. 2007; 1: 016001.

29. Grote C, Pawliszyn J. Solid-phase microextraction for the analysis of human breath. Anal Chem. 1997; 69: 587-96.

30. Zhang JJ, Gu MM, Zheng TT, et al. Synthesis of gelatin-stabilized gold nanoparticles and assembly of carboxylic single-walled carbon nanotubes/ $\mathrm{Au}$ composites for cytosensing and drug uptake. Anal. Chem. 2009; 81: 6641-6648.

31. Bali PB, Jauhari D, Prasad Tiwari M. A dual-template imprinted polymer-modified carbon ceramic electrode for ultra-trace simultaneous analysis of ascorbic acid and dopamine. Biosens Bioelectron. 2013; 50C:19-27.

32. Espinosa E, Ionescu R, Bittencourt C, et al. Metal-decorated multi-wall carbon nanotubes for low temperature gas sensing. Thin Solid Films. 2007; 515: 8322-27.

33. Chen DP, Zhu G, Zhu XG, et al. Effects of silver nanowires on the electrochemical performance of LiFePO4. Nano Biomed Eng 2011; 3: 19-24.

34. Hu CY, Yang DP, Wang ZH, et al. Bio-mimetically synthesized Ag@BSA microspheres as a novel electrochemical biosensing interface for sensitive detection of tumor cells. Biosens.Bioelectron.2013; 41: 656-662.

35. Cui DX, Pan BF, Zhang H, et al. Self-assembly of quantum dots and carbon nanotubes for ultrasensitive DNA and antigen detection. Anal. Chem. 2008; 80: 7996-8001.

36. Gui C, Dai X, Cui DX. Advances of Nanotechnology Applied to Biosensors. Nano Biomed. Eng. 2011; 3: 260-273.

37. Liu HY, Shen GX . Ordered arrays of carbon nanotubes: from synthesis to applications. Nano Biomed. Eng. 2012; 4: 107-117.

38. Zhao X, Zhang B, Ai K, et al. Monitoring catalyticdegradation of dye molecules on silver-coated $\mathrm{ZnO}$ nanowire arrays by surface-enhanced Raman spectroscopy. J Mater Chem. 2009; 19: 5547-53.

39. Ma Y, Ali SR, Dodoo AS, et al. Enhanced sensitivity for biosensors: multiple functions of DNA-wrapped single-walled carbon nanotubes in self-doped polyaniline nanocomposites. J of Phys Chem B. 2006; 110: 16359-16365.

40. Zhao Q, Nardelli MB, Lu W, et al. Carbon nanotube-metal cluster composites: A new road to chemical sensors? Nano Lett. 2005; 5: 847-851.

41. Mallin MP, Murphy CJ. Solution-phase synthesis of sub-10 nm Au-Ag alloy nanoparticles. Nano Lett. 2002; 2: 1235-1237. 
42. Shibata T, Bunker BA, Zhang Z, et al. Size-dependent spontaneous alloying of Au-Ag nanoparticles. J Am Chem Soc. 2002; 124: 1989-1996.

43. Sun L, Luan WL, Tu ST, et al. Au-Ag gradient alloy nanoparticles with extended surface plasmon resonance wavelength: synthesis via microreaction. Nano Biomed. Eng.2011; 3: 232-235.

44. Valden M, Lai X, Goodman DW. Onset of catalytic activity of gold clusters on titania with the appearance of nonmetallic properties. Science 1998; 281: 1647-1650.

45. Feng LL, Gao G, Huang P, et al. Optical properties and catalytic activity of bimetallic gold-silver nanoparticles. Nano Biomed Eng 2010; 2: 258-267.

46. Schomburg I, Hofmann O, Baensch $\mathrm{C}$, et al. Enzyme data and metabolic information: BRENDA, a resource for research in biology, biochemistry, and medicine. Gene Function \& Disease. 2000; 3: 109-118.

47. Cui DX. Advances and prospects on biomolecules functionalized carbon nanotubes. Journal of Nanoscience and Nanotechnolgy, 2007; 7: 1298-1314.

48. Shin HW, Umber BJ, Meinardi S, et al. Acetaldehyde and hexanaldehyde from cultured white cells. J Transl Med. 2009; 7: 1-11.

49. Patel M LL, Zander DS, Sreerama L, et al. ALDH1A1 and ALDH3A1 expression in lung cancers: Correlation with histologic type and potential precursors. Lung Cancer. 2008;59:340-349.

50. Miodek A, Castillo G, Hianik T, et al. Electrochemical aptasensor of human cellular prion based on multiwalled carbon nanotubes modified with dendrimers: A platform for connecting redox markers and aptamers. Anal. Chem. 2013; 85:7704-7712. 